\title{
A Unique Presentation of Spontaneous Compartment Syndrome due to Acquired Hemophilia A and Associated Malignancy: Case Report and Literature Review
}

\author{
Marjan Alidoost ${ }^{\mathrm{a}, \mathrm{b}}$, Gabriella A. Conte ${ }^{\mathrm{a}}$, Rabhea Chaudry ${ }^{\mathrm{a}}$, \\ Kenneth Nahum ${ }^{\mathrm{a}}$, Diane Marchesani ${ }^{\mathrm{a}}$
}

\begin{abstract}
Hemophilia is a bleeding diathesis that is most commonly congenital and causes a tendency for significant bleeding during procedures and often manifests as hemarthrosis. However, more rarely, hemophilia can be acquired. Our paper focuses on acquired hemophilia A (AHA), which is caused by the development of an autoantibody (an inhibitor) to factor VIII. A 61-year-old man with a past medical history of type II diabetes mellitus, hypertension, hyperlipidemia, hypothyroidism, and obstructive sleep apnea presented to the emergency department with severe right lower extremity pain and swelling of 2-day duration. He was found to have compartment syndrome and underwent emergent fasciotomy of his right leg. After surgery he still had significant bleeding, despite transfusions and administration of fresh frozen plasma (FFP) by the surgical team. He was later diagnosed with AHA, but was not adequately responsive to factor VII, factor VIII, steroids nor rituxan and unfortunately had his right lower extremity amputated. He had a prolonged hospital course, which included Streptococcus bovis bacteremia and a code stroke for which head computed tomography (CT) showed probable metastasis. It was acknowledged he had probable metastatic colon cancer, which was not confirmed as the patient transitioned to hospice care. Rather than hemarthrosis, patients with AHA tend to have bleeding in soft tissue or the gastrointestinal tract. AHA can have underlying causes, such as malignancy. AHA associated with malignancy is associated with poorer outcomes and tends to improve with treatment of the underlying malignancy. Therefore, it is important to quickly identify these patients and screen them for underlying etiologies.
\end{abstract}

Keywords: Acquired hemophilia A; Spontaneous compartment syndrome; Colon carcinoma; Streptococcus bovis; Malignancy; Case report

Manuscript submitted January 14, 2020, accepted February 14, 2020

aDepartment of Medicine, Jersey Shore University Medical Center, Hackensack Meridian Health, Neptune, NJ 07753, USA

${ }^{b}$ Corresponding Author: Marjan Alidoost, Department of Medicine, Jersey Shore University Medical Center, Hackensack Meridian Health, Neptune, NJ 07753, USA. Email: Marjan.Alidoost@hackensackmeridian.org

doi: https://doi.org/10.14740/wjon1260

\section{Introduction}

Acquired hemophilia A (AHA) is a potentially severe lifethreatening bleeding diathesis caused by the spontaneous development of autoantibodies against naturally derived coagulation factors. AHA is a very rare condition with an approximate incidence of 1.48/million/year [1]. Factor (F)VIII, an X-linked gene product, is an important catalyst in the coagulation cascade; FVIII contributes to secondary hemostasis via the intrinsic pathway by combining with FIXa, calcium and phospholipids to activate factor X [2]. Absence or reduced levels of the FVIII gene (due to mutation, deletion, and inversion) is referred to as classic hemophilia A, an X-linked recessive congenital disorder that predominantly affects male children $[3,4]$. Conversely, the condition in which autoantibodies, immunoglobulin $\mathrm{G}$ ( $\mathrm{IgG})$ oligoclonal or restricted polyclonal antibodies, are formed against and inactivate the FVIII coagulant protein is referred to as AHA, which occurs in a biphasic age distribution, commonly affecting men and women over 60 years of age $(>80 \%)[1,5]$. While both acquired and inherited conditions involve deficient FVIII function and activity, their clinical features differ. Bleeding episodes in AHA typically occur spontaneously and manifest as subcutaneous or deep soft tissue hematomas, gastrointestinal or urinogenital bleeding, whereas hemarthroses constitute $70-80 \%$ of the bleeding episodes in congenital hemophilia patients $[4,6,7]$.

AHA is a rare disorder, with an incidence of 1 to 4 cases per million per year in the non-hemophilic population [2]. In $30-40 \%$ of AHA cases, common underlying etiologies include autoimmune diseases (14.1\%), malignancy (11.5\%), drugs (such as phenytoin and penicillin) and pregnancy and postpartum period (8.9\%) [8]. A recent systematic literature review reports a large number of AHA patients with solid cancers, with the most common being prostate cancer $(25.3 \%)$, lung cancer $(15.8 \%)$, followed by colon cancer $(9.5 \%)$ [8]. AHA in association with malignancy has been described in several case reports, including gastric, hepatocellular, thyroid, breast and lung carcinomas [9]. In this paper, we describe a rare case of probable colorectal carcinoma detected in a patient with AHA. 
Table 1. Laboratory Values on Admission

\begin{tabular}{lll}
\hline Laboratory measure & Reference range & Value \\
\hline Hemoglobin & $13.2-17.5 \mathrm{~g} / \mathrm{dL}$ & 7.7 \\
Hematocrit & $40.0-53.0 \%$ & 25.7 \\
White blood cell count & $4.5-11.0 \mathrm{~K} / \mu \mathrm{L}$ & 7.9 \\
Platelets & $140-450 \mathrm{~K} / \mu \mathrm{L}$ & 279 \\
Bilirubin & $0.2-1.3 \mathrm{mg} / \mathrm{dL}$ & 1.4 \\
Alkaline phosphatase & $38-126 \mathrm{IU} / \mathrm{L}$ & 69 \\
AST & $10-42 \mathrm{IU} / \mathrm{L}$ & 28 \\
ALT & $10-60 \mathrm{IU} / \mathrm{L}$ & 17 \\
INR & $0.88-1.15$ & 1.31 \\
aPPT & $26-39 \mathrm{~s}$ & 44 \\
Factor VIII assay & $50-150 \%$ & 5.6 \\
Factor VIII inhibitor & $<0.5 \mathrm{units}$ & 14.6 \\
\hline
\end{tabular}

AST: aspartate transaminase; ALT: alanine transaminase; INR: international normalized ratio; aPPT: activated partial prothrombin time.

\section{Case Report}

A 61-year-old man with a past medical history of type II diabetes mellitus, hypertension, hyperlipidemia, hypothyroidism, and obstructive sleep apnea presented to the emergency department (ED) with right lower extremity pain and swelling. His pain began 2 days prior to admission and was initially mild and only behind his knee. Of note, he had noticed increased bruising and was seen by hematology/oncology and vascular surgery outpatient who diagnosed him with B12 and iron deficiency. At home he took levothyroxine $137 \mu \mathrm{g}$ once daily in the morning (QAM), norvasc $5 \mathrm{mg}$ daily, and aspirin $81 \mathrm{mg}$ daily. $\mathrm{He}$ did not have any significant social or family history. He had not had any recent falls or trauma. He came to the ED after his pain worsened to the point that he could not bear weight on his leg and he developed paresthesia and numbness of his toes. He was evaluated by vascular surgery and determined to have compartment syndrome of the posterior compartment. He then went for emergent fasciotomy of his right lower extremity posterior compartment on the day of admission. Postoperatively he continued to have bleeding and received seven units of packed red blood cells (pRBCs) and fresh frozen plasma (FFP). The next day a rapid response was called for diaphoresis, weakness, and right lower extremity pain. He was evaluated by orthopedics and subsequently transferred to the surgical intensive care unit for hemorrhagic shock. At that time, he received three additional units of pRBCs. He went for repeat fasciotomy the following day and was found to be hemorrhaging in his right lower extremity posterior compartment.

Laboratory values from his admission showed a prolonged activated partial prothrombin time (aPPT) (Table 1). On his second day of hospitalization, hematology/oncology was consulted and, based on his laboratory values, it was determined that he had a coagulopathy due to suspected FVIII inhibition and he was administered both FVIII and steroids. Hematology/oncology then ordered FVIII levels which later returned as markedly decreased (Table 1). Bethesda inhibitor test was also done which was positive for FVIII inhibitor levels (Table 1). His bleeding was not controlled by the combination of FVIII and steroids and thus he was given a trial of recombinant FVII with steroids which, initially, controlled the bleeding of his right lower extremity, but was ultimately not successful. At this time, he remained hemodynamically stable but became blood transfusion dependent. Over the course of 6 weeks he was also trialed on recombinant porcine FVIII, rituximab, intravenous immunoglobulin (IVIG), and plasmapheresis. Of note, during his first 4 weeks of hospitalization he had a total of seven debridements of his right lower extremity in attempt to control his persistent hemorrhaging. However, during his fifth week of hospitalization, he underwent right lower extremity amputation above the knee.

During his sixth week of hospitalization he developed new-onset progressive thrombocytopenia; his platelets rapidly dropped to $32 \mathrm{~K} / \mu \mathrm{L}$. Due to high concern for bacteremia blood cultures were collected and resulted positive for Streptococcus bovis and Escherichia coli. At this time the patient was seen by infectious disease doctor who initiated intravenous ceftriaxone. Colonoscopy was recommended but due to his bleeding risk from AHA and worsening thrombocytopenia it was postponed upon gastrointestinal evaluation. It was suspected that his positive cultures were due to probable gastrointestinal translocation.

On his 11th week of hospitalization a code stroke was called for acute right-sided weakness and altered mental status. Computed tomography (CT) of the head was performed and showed multiple hypodensities suspicious for stroke versus possible metastasis. Subsequent magnetic resonance imaging (MRI) of the brain showed innumerable peripheral enhancing mass lesions, highly suspicious for multifocal metastases (Fig. 1). He was ultimately intubated for airway protection and sent to the neurological intensive care unit. Due to high suspicion for malignancy he had an abdomen/pelvis CT which was negative for a mass. As the patient's mental status did not improve, his family met with palliative care and his code status was changed to "do not resuscitate". During this same week, he had a pulseless electrical activity (PEA) cardiac arrest and expired.

\section{Discussion}

Acquired FVIII inhibitor leads to AHA, which does not typically present as classic congenital hemophilia [10]. Congenital hemophilia presents in children with hemarthrosis while AHA presents in adults, typically 60 or older, and tends to have soft tissue bleeding rather than hemarthrosis [10]. AHA has also been reported to present as gastrointestinal bleeding, retroperitoneal bleeding, epistaxis, post-partum bleeding, and iatrogenic bleeding that is of sudden and severe onset [10]. AHA has less commonly been reported to present as spontaneous compartment syndrome, as with our patient [11]. The bleeding associated with AHA is usually more severe, with an estimated $90 \%$ of patients having severe bleeding and there is an estimated mortality rate of $8-22 \%$ [10]. Patients with AHA have a long activated partial thromboplastin time (aPTT) that does not correct with mixing studies due to the presence of the 


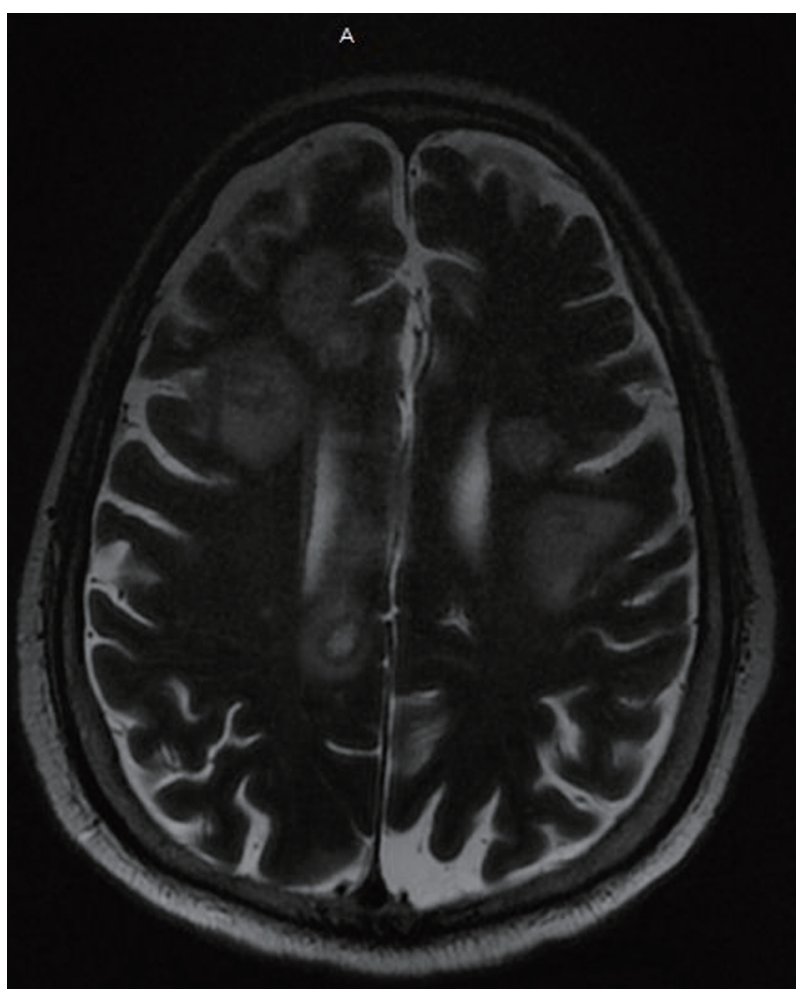

Figure 1. MRI of the brain with and without contrast shows innumerable peripheral enhancing mass lesions highly suspicious for multifocal metastases. MRI: magnetic resonance imaging.

eponymous FVIII inhibitor. AHA can be diagnosed with FVIII inhibitor titer levels with a Bethesda study to make the formal diagnosis [11].

Not only is it important for the physician to have a high suspicion for AHA in patients with isolated prolonged aPTTs without explanation, but also to be aware that $30-40 \%$ of reported cases of AHA have an underlying cause [8]. There is a myriad of causes of AHA, ranging from autoimmune (such as systemic lupus erythematous) to drug-related to pregnancyrelated to malignancy-related [10]. Malignancy remains a significant cause of AHA with an estimated $10-15 \%$ of patients with AHA having cancer as their underlying diagnosis [12]. AHA has been reported with solid and hematologic malignancies and has a worse prognosis with solid malignancies as they are typically detected at a later stage [9]. It is not classically associated with any one cancer, such as the association of Lambert-Eaton myasthenia syndrome with small-cell lung carcinoma, but is most commonly reported in association with lung and prostate cancer [9]. Notably, there is an association with AHA secondary to malignancy and higher rates of recurrent and severe disease $[8,9]$. In patients who are found to have treatment-resistant AHA, it is important for the clinician to investigate for underlying malignancy. Patients with AHA secondary to cancer who received treatment for their cancer had an $88.8 \%$ global success rate [8]. Conversely, patients with more advanced malignancy had higher rates of treatmentresistant disease and a worse prognosis [8]. Additionally, in patients who have underlying solid malignancies, such as our patient, malignancies are usually detected at more advanced stages. These patients thus have a worse prognosis than those with hematologic malignancies [9]. It is currently not understood what causes the pathogenesis of AHA in patients with cancer, although it has been theorized to be due to immune system alteration in cancer patients or as a potential sequalae of chemotherapy [9].

There are three steps to treat AHA associated with malignancy. First, one must stop the bleeding and stabilize hemostasis which can be done using various FVIII bypassing agents such as recombinant FVII, FVIII inhibitor bypassing activity (FEIBA), and activated prothrombin complex concentrate (aPCC) [10]. The EACH2 trial showed increased efficacy of bypassing agents compared to FFP, 1-deamino-8-D-arginine vasopressin (DDAVP), and porcine FVIII [5]. It is also important to avoid unnecessary procedures as even traditionally low risk procedures have a risk of future insult and hemorrhage [8]. The second step is to decrease the amount of inhibitor, which can be done with various immunosuppressant agents, namely corticosteroids in combination with another agent [10]. The agent that has been most studied and is considered as first line is cyclophosphamide along with steroids [10]. Corticosteroids and rituximab are considered as second line, with steroids combined with cyclophosphamide having an estimated success rate of $70 \%$ versus steroids combined with rituximab having an estimated success rate of $58 \%$ [8]. The third step is to treat the underlying malignancy. For patients with resistant disease, there have been limited studies on treating with plasmapheresis and immunoadsorption, but it is unclear how efficacious plasmapheresis and immunoadsorption are in treating AHA with malignancy [8]. Our patient, prior to his diagnosis of cancer but after many trials of immunosuppressive agents, was trialed on plasmapheresis which did not control his disease. Unfortunately, our patient's bleeding was difficult to stabilize, and he ultimately had a poor outcome.

Our patient's presumed diagnosis of colon carcinoma was not confirmed via biopsy but was highly suspected due to positive Streptococcus bovis bacteremia and its known association with colon carcinoma. His head CT and MRI were highly suspicious for metastases, which likely contributed to his poor outcome. Upon performing a PubMed literature search there is one similar case report of a 63-year-old woman who presented with AHA and who was ultimately diagnosed with sigmoid adenocarcinoma [12]. Initial hemostasis stabilization was achieved with recombinant FVII. Attempt to decrease FVIII inhibitor with steroids and cyclophosphamide failed but was ultimately attained with steroids and rituximab [12]. The patient reported by Ichikawa et al conversely was not found to have metastatic disease; as it was believed our patient had it, he eventually underwent a colectomy and achieved complete remission.

\section{Conclusions}

In conclusion, we present a rare presentation of AHA manifesting as spontaneous compartment syndrome due to probable underling colon carcinoma. Compared to congenital hemophilia patients who present with hemarthrosis, AHA patients 
generally present as gastrointestinal or retroperitoneal bleeding which is more severe, difficult to control and associated with higher mortality rates. Additionally, malignancy has been reported in the literature as a potential underlying etiology of AHA. We thus suggest that malignancy should be included in the differential diagnosis and active workup in patients with suspected AHA, as the clinical course tends to improve with treatment of the underlying etiology. Identification of an underlying cause of AHA is important to facilitate timely and appropriate treatment.

\section{Acknowledgments}

The authors gratefully acknowledge the support of the Departments of Infectious Disease and Oncology at Jersey Shore University Medical Center, Hackensack Meridian Health. We thank our patient who kindly gave his consent for this publication.

\section{Financial Disclosure}

This project was not supported by any grants or funding agencies.

\section{Conflict of Interest}

The authors declare that there is no conflict of interest regarding the publication of this paper.

\section{Informed Consent}

Written informed consent was obtained from the patient for publication of this case report and any accompanying images.

\section{Author Contributions}

Each author was involved and participated in drafting the manuscript and revising it critically for important intellectual content and gave final approval of the version to be published. Each author agreed to be accountable for all aspects of the work in ensuring that questions related to the accuracy or integrity of any part of the work are appropriately investigated and resolved. All authors are aware of and approve the manuscript being submitted for publication. DM and $\mathrm{KN}$ encouraged MA, GAC, and RC to learn about acquired hemophilia A and underlying malignancy. DM presented the idea. MA, GAC and $\mathrm{RC}$ wrote the manuscript with input from all authors. MA and GAC finalized the manuscript.

\section{Data Availability}

All data generated or analyzed during this study are included in this article.

\section{References}

1. Collins PW, Hirsch S, Baglin TP, Dolan G, Hanley J, Makris M, Keeling DM, et al. Acquired hemophilia A in the United Kingdom: a 2-year national surveillance study by the United Kingdom Haemophilia Centre Doctors' Organisation. Blood. 2007;109(5):1870-1877.

2. Shumail S, Junaid A, Rashid K. Acquired haemophilia due to factor-VIII inhibitors secondary to bladder malignancy: a case report. Int J Pathol. 2016;14(1).

3. DiMichele DM. Inhibitors in haemophilia: a primer. Haemophilia. 2000;6(Suppl 1):38-40.

4. Giangrande P. Acquired hemophilia. Treatment of Hemophilia. World Federation of Hemophilia. 2012;38:1-5.

5. Baudo F, Collins P, Huth-Kuhne A, Levesque H, Marco P, Nemes L, Pellegrini F, et al. Management of bleeding in acquired hemophilia A: results from the European Acquired Haemophilia (EACH2) Registry. Blood. 2012;120(1):39-46.

6. Srivastava A, Brewer AK, Mauser-Bunschoten EP, Key NS, Kitchen S, Llinas A, Ludlam CA, et al. Guidelines for the management of hemophilia. Haemophilia. 2013;19(1):e1-47.

7. Collins P, Baudo F, Huth-Kuhne A, Ingerslev J, Kessler $\mathrm{CM}$, Castellano ME, Shima M, et al. Consensus recommendations for the diagnosis and treatment of acquired hemophilia A. BMC Res Notes. 2010;3:161.

8. Napolitano M, Siragusa S, Mancuso S, Kessler CM. Acquired haemophilia in cancer: A systematic and critical literature review. Haemophilia. 2018;24(1):43-56.

9. Saito M, Ogasawara R, Izumiyama K, Mori A, Kondo T, Tanaka M, Morioka M, et al. Acquired hemophilia A in solid cancer: Two case reports and review of the literature. World J Clin Cases. 2018;6(14):781-785.

10. Franchini M, Lippi G. Acquired factor VIII inhibitors. Blood. 2008;112(2):250-255.

11. Pham TV, Sorenson CA, Nable JV. Acquired factor VIII deficiency presenting with compartment syndrome. Am J Emerg Med. 2014;32(2):195 e191-192.

12. Ichikawa S, Kohata K, Okitsu Y, Suzuki M, Nakajima $\mathrm{S}$, Yamada MF, Onishi Y, et al. Acquired hemophilia A with sigmoid colon cancer: successful treatment with rituximab followed by sigmoidectomy. Int $\mathrm{J}$ Hematol. 2009;90(1):33-36. 